\title{
Reliability based Maintenance Model to Assess the Condition of Rotor using XLrotor
}

\author{
Murgayya S B ${ }^{\mathrm{a},{ }^{*}}$, H N Suresh ${ }^{\mathrm{a}}$, Madhusudhan $\mathrm{N}^{\mathrm{b}}$, and Sarvanabavan $\mathrm{B}^{\mathrm{c}}$ \\ ${ }^{a}$ Department of Automobile Engineering, Dayananda Sagar College of Engineering, Bengaluru, 560078, India \\ ${ }^{b}$ ISO CAT-2 Vibration Analyst, Diagnostic Engineers, Bengaluru, 560078, India \\ ${ }^{c}$ School of Mechanical Engineering, Dayananda Sagar University, Bengaluru, 560078, India
}

\begin{abstract}
The work carried out is focused on study and behavior of rotors and to check the reliability at the design stage using the advanced XLrotor tool. For the selected rotor, the failures are controlled by shifting critical speeds above the maximum speed in which it is designed for. The rotor is balanced with safe limits of vibration severity criteria as per ISO standards for an optimized model. Sensitivity analysis is carried out on the rotor model by varying factors such as the diameter of shaft, disk configuration, disk offset, and load on rotor model. The results obtained show good agreement with the FEM results. The results obtained are undamped \& damped critical speeds, as well as velocity \& dynamic load on bearings due to imbalance using XLrotor. The rotor model is balanced for a steel shaft with a steel disk as well as a steel shaft with an aluminum disk (optimized rotor model) as part of the corrective method. The work clearly emphasizes XLrotor for designing a highly reliable rotor that is safe for operating conditions.
\end{abstract}

Keywords: reliability; sensitivity analysis; rotor dynamics \& XLrotor

(Submitted on July 16, 2019; Revised on August 16, 2019; Accepted on August 29, 2019)

(C) 2019 Totem Publisher, Inc. All rights reserved.

\section{Introduction}

In rotor dynamics, depending on the structure and mechanism involved, a machine produces vibration signatures. These machine vibrations can increase and excite the natural frequency of the rotor and cause resonance. The effect of failure of the rotor depends on various influencing factors i.e mass, stiffness, material configuration, disk offset, disk configuration, bearing stiffness and damping properties. A rotating system's critical speed occurs when a natural frequency matches its rotational speed. To reduce the overall forces that initiate resonance, it is important to minimize rotational unbalance and unnecessary external forces [1]. This paper highlighted on reliable rotor dynamic design as a whole, ranging from systematic R\&D efforts to high-demand testing [2]. The literature shows several examples of high sub synchronous vibrations that made necessary changes to the machine [3-5]. This work focuses on emphasizing some of the latest findings on rotating machine common defects. The selected faults include misalignment, unbalance, looseness, instability, bearing failures, shaft cracks, crack blades and shaft bow. Each of these defects were detailed in terms of sensors, fault identification techniques, location, modeling and prognosis. At the initial stage, the rotor is designed and analyzed to its intended function. On-site problems arise due to various factors. One of the main causes of rotor failure is due to imbalance. There is an inherent degree of unbalance in each rotating machine [6]. The analysis on 1 DOF Jeffcott rotor with disk offset illustrates a detailed study on an analytical model and simulation method, which gives a fundamental idea on disk offset of complex rotors. In a study of rotordynamics the failure of rotor occurs where critical speeds will vary due to disk offset [7]. Work summaries on condition monitoring and recent trends in the area of rotor dynamics was done by using cloud computing network technology and an analytical model [8]. In this work, the rotordynamics analysis using the FEM method is highlighted through features and benefits with gyroscopic effects, which affects the critical speeds. The design and testing of a compulsory rotor model scale has been described and during rotor balancing, the valuable experience of a single plane and multi plane were gained. The rotor was first balanced on a commercial balancing machine to extreme precision at low speeds [9]. The main aim in this paper is to find a methodology for system identification using analytical techniques and 
update the model using experimental techniques for active rotating rigid structures, which subsequently contributes to the implementation of health monitoring and further design and development of rotating machinery [10]. Firstly, gyroscopic moments tend to combine dynamics is two radial movement directions. Along the horizontal dynamics, the rotor gets affected due to changes in the vertical state of the rotor and vice versa. Secondly, gyroscopic moments cause the system's critical speeds to drift at zero speed from their original position. As a function of rotational speed, the gyroscopic moment acting on a rotor may increase or decrease the critical speeds associated with certain system modes [11]. The Dyrobes simulation model has greatly influenced the method of analysis of the Jeffcott rotor and its effects on critical speeds. By the analytical and simulation method, the model is studied and analyzed for the Jeffcott rotor and it shows the features and benefits of the simulation method. The torsional vibrations problems are discussed and analyzed to solve serious machine problems through experimental technique [12]. Fatigue crack development can have extremely damaging effects on the reliability of rotating machinery with enormous rotating masses. The work focused on the detection of cracks in the rotor. It can be concluded that significant changes in parameters that describes the rotor's oscillatory behavior during the run-up were noted as a result of rotor crack development [13]. In carrying out vibration measurement and analysis, an effort should be made to collect effective data. Depending on the speed of the rotor, the vibration is measured on the following factors 1) Slow speed - Displacement as failures are due to stress concentration 2) Medium speed - Velocity as failures are due to fatigue \& 3) High speed - Acceleration as failures are due to excessive force.

\section{Modeling and Simulation}

For selecting the model, the analysis is carried out in XLrotor to shift the critical beyond its maximum speed and for safe and high reliability. The analysis of the rotor is determined by varying diameters of the shaft, disk configuration, disk offset and load on disk. The testing trails highlight undamped and damped critical speeds, velocity of rotor, and load on bearing due to the imbalance of $200 \mathrm{gm}-\mathrm{mm}$ [14]. An attempt is made to reduce vibration of rotor through balancing. The rotor model and disk properties are as shown in Figure 1 and Table 1:

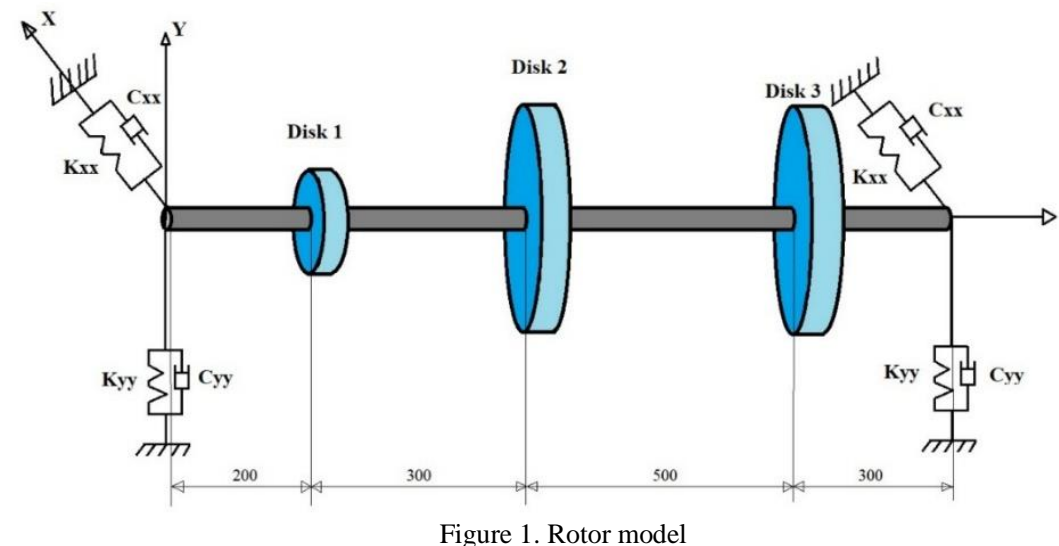

Table 1. Disk properties

\begin{tabular}{|c|c|c|c|c|c|}
\hline Disk & OD in $\mathrm{mm}$ & $t$ in $\mathrm{mm}$ & Density $\mathrm{kg} / \mathrm{m}^{3}$ & Imbalance mass gm-mm & Position in $\mathrm{mm}$ \\
\hline 1 & 240 & 50 & 7800 & 0 & 200 \\
\hline 2 & 400 & 50 & 7800 & 200 & 500 \\
\hline 3 & 400 & 60 & 7800 & 0 & 1000 \\
\hline
\end{tabular}

The rotor model length is limited to $1300 \mathrm{~mm}$ and the diameter of the shaft to $100 \mathrm{~mm}$. For the model, these subcomponents include the shaft or the rotor, built-on parts, and the support system. The built-on parts include impellers, gears, disks, fans, couplings and any other part that rotates with the shaft can be attached to the rotor.

The rotor is divided into 27 stations as shown in Figure 2, with an increase in the number of divisions of the rotor model. In the shaft input sheet, the accuracy of the results also increases. Station 1 and Station 27 represent bearing locations. Two identical bearings are placed at the ends of the rotor with the following stiffness and damping coefficients [14].

$$
\begin{aligned}
& K_{x x}=5.0 \times 10^{7} \mathrm{~N} / \mathrm{m}, C_{x x}=5.0 \times 10^{2} \mathrm{~N}-\mathrm{s} / \mathrm{m} \\
& K_{y y}=7.0 \times 10^{7} \mathrm{~N} / \mathrm{m}, C_{y y}=7.0 \times 10^{2} \mathrm{~N}-\mathrm{s} / \mathrm{m}
\end{aligned}
$$




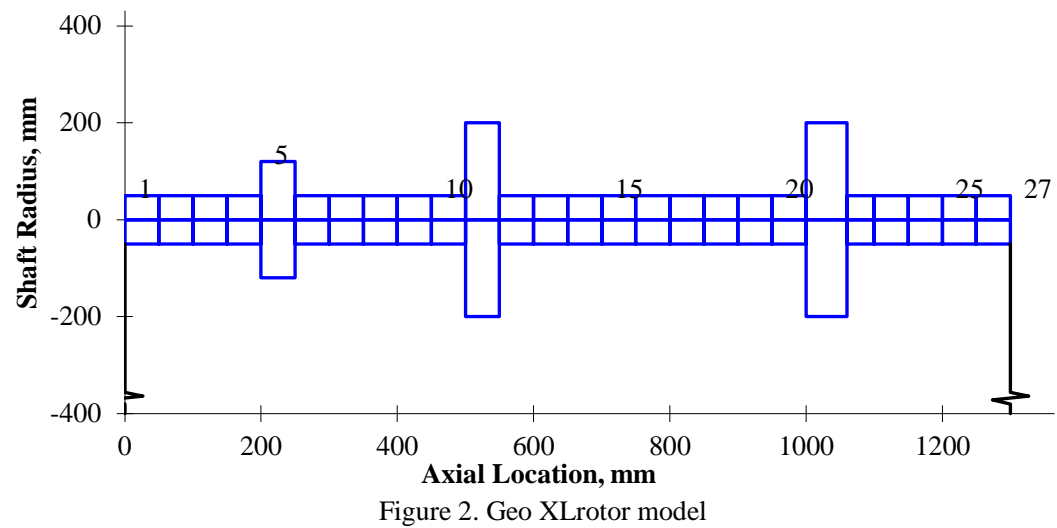

These values are typical of stiffness and damping for fluid film bearings. However, these values are independent of running speed, unlike fluid film bearings [14-15].

It is clearly observed that the bearing stiffness directly affects the rotor critical speed (shown in Table 2). The value $1000000 \mathrm{~N} / \mathrm{m}$ represents that the model is supported by soft flexible bearings and for $3162277660 \mathrm{~N} / \mathrm{m}$ rigidly supported bearings. The limiting value of stiffness is based on the applications and functions of rotor. This analysis represents the model from free to rigidly supported and checks whether the rotors are intentionally constrained or not. Figure 3 shows quick look on undamped critical speeds vs bearing stiffness.

Table 2. Undamped critical speeds in cycles per minute (cpm) vs bearing stiffness $\mathrm{N} / \mathrm{m}$

\begin{tabular}{|c|c|c|c|c|}
\hline \multirow{2}{*}{ Stiffness (N/m) } & \multicolumn{4}{|c|}{ Undamped critical speeds in cpm } \\
\cline { 2 - 5 } & 1 & 2 & 3 & 4 \\
\hline 1000000. & 945.9 & 1914.8 & 15481.8 & 54612.4 \\
\hline 3162278. & 1620.7 & 3370.6 & 16014.2 & 55077.3 \\
\hline 10000000. & 2597.2 & 5809.6 & 17587.4 & 56519.4 \\
\hline 31622777. & 3636.3 & 9446.7 & 21663.3 & 60812.3 \\
\hline 100000000. & 4331.5 & 13544.9 & 29687.2 & 72153.5 \\
\hline 316227766. & 4640.3 & 16473.1 & 39750.8 & \\
\hline 1000000000. & 4750.8 & 17831.4 & 46561.2 & \\
\hline 3162277660. & 4787.2 & 18326.3 & 49375.3 & \\
\hline \multicolumn{4}{|c}{} \\
\hline
\end{tabular}

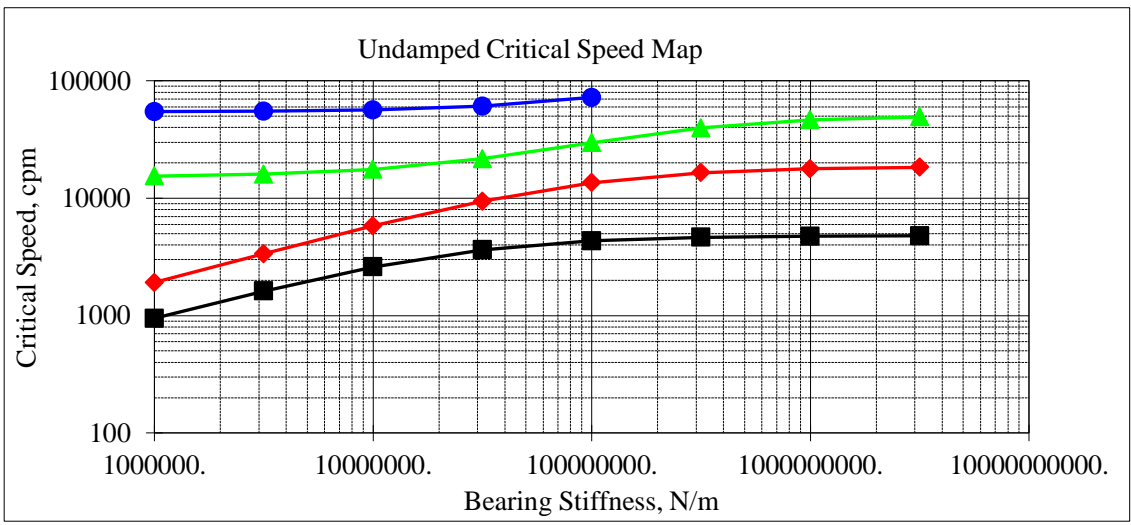

Figure 3. Critical speeds vs bearing stiffness

The results obtained from XLrotor in Table 3 and Figure 4 show a critical speed at $3900 \mathrm{cpm}$, which is congruent with FEM results.

Table 3. Damped critical speeds

\begin{tabular}{|c|c|c|c|}
\hline \multirow{2}{*}{ Modes } & \multicolumn{3}{|c|}{ Damped critical speeds, cpm } \\
\cline { 2 - 4 } & FEM [16] & XLrotor & \% Error \\
\hline 1 & 3757 & 3900 & 3.8 \\
\hline 2 & 10947 & 10618 & 3.0 \\
\hline
\end{tabular}




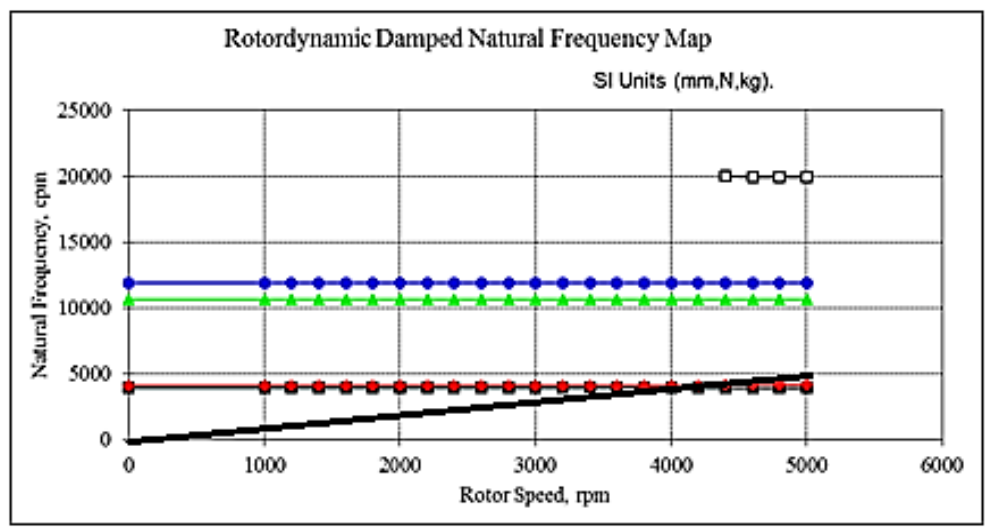

Figure 4. Damped critical speeds with synchronous

A precise benefit of solid models is the presence of spin softening, stress stiffening and temperature effects in the rotor model analysis is considered in this conventional modelling. The effect of spin softening has a significant influence on the modes of backward whirl and the effect of stress stiffening on the modes of forward whirl in this rotor. It is shown how these parameters affect rotor performance and causes resonance due to synchronous mode. The variation of stress stiffening and soft stiffening effects depends on geometrical model, material selection and boundary conditions. Since an intentional imbalance is located on disk 2 i.e. $200 \mathrm{gm}-\mathrm{mm}$ [14], the vibration of the rotor due to imbalance is determined through XLrotor, which shows the $1^{\text {st }}$ critical speed at $3905(65 \mathrm{~Hz})$. The vibration at station 11 is extremely high, which is vibrating with a velocity of $0.25 \mathrm{~m} / \mathrm{s}$ at $3900 \mathrm{cpm}$ in the horizontal amp, and $0.09 \mathrm{~m} / \mathrm{s}$ at $4100 \mathrm{cpm}$ in the vertical amp respectively. Figure 5 shows the rotordynamic response plot.

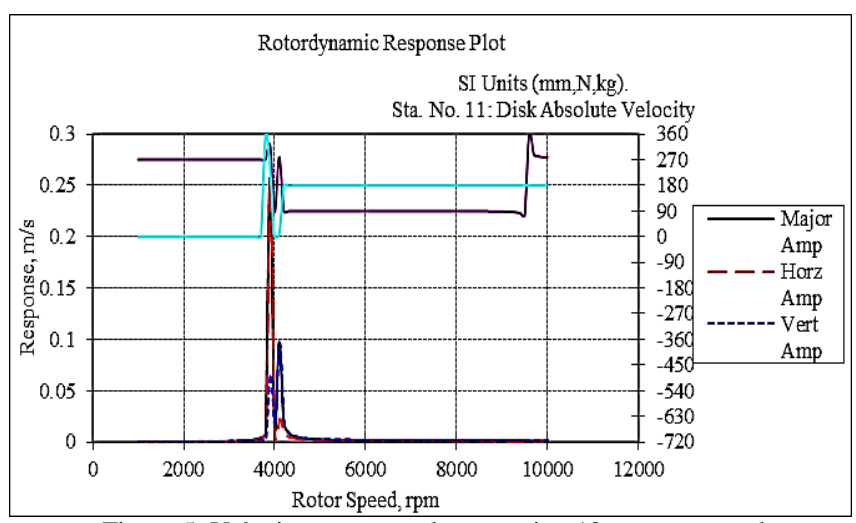

Figure 5. Velocity response plot at station 12 vs rotor speed

The vibrations are extremely high compared to the ISO Vibration Severity Criteria (VSC), which causes resonance and leads to catastrophic failure of the rotors and bearings. When rotors are designed for high speed, it is mandated to calculate the load acting on bearings due to imbalance. Since the rotor is vibrating at high speeds, the load on both fluid bearings is as shown in Figures 6(a) and 6(b).

From Figures 6(a) and 6(b), the results obtained show good agreement to the vibration trouble shooting chart (nature of imbalance fault, the frequency of dominant vibration and direction in radial) i.e. the load acting on this bearing is governing radial direction. As vibration velocity at station 11 is high, correspondingly load acting on bearings is also high in the horizontal direction. The bearings at station 27 will fail earlier than station 1, as an additional mass of $31.36 \mathrm{~kg}$ on disk 2 and $41.16 \mathrm{~kg}$ on disk 3 is distributed towards station 27 on the rotor. This is also because the centre of gravity is not at the centre of the disk; it is in between disk 2 and disk 3. In order to shift critical speeds, the sensitivity analysis of the rotor model is carried out as shown in 2.1 .

\subsection{Sensitivity Analysis of Rotor}

The main objective of this work is to design the rotor for safe operating conditions and to achieve high reliability. This can be achieved when rotor critical speeds, vibration, and load on bearings are shifted beyond its maximum speed. Hence, to achieve this, an attempt is made on the sensitivity analysis of the rotor. The results will determine the safe and reliable 
conditions of the rotor. For future development, this work gives a glimpse on rotor design in XLrotor. The sensitivity analysis on the rotor is carried out but not limited to 1) Diameter of shaft; 2) Disk configuration; 3) Disk offset; 4) Load on disk.

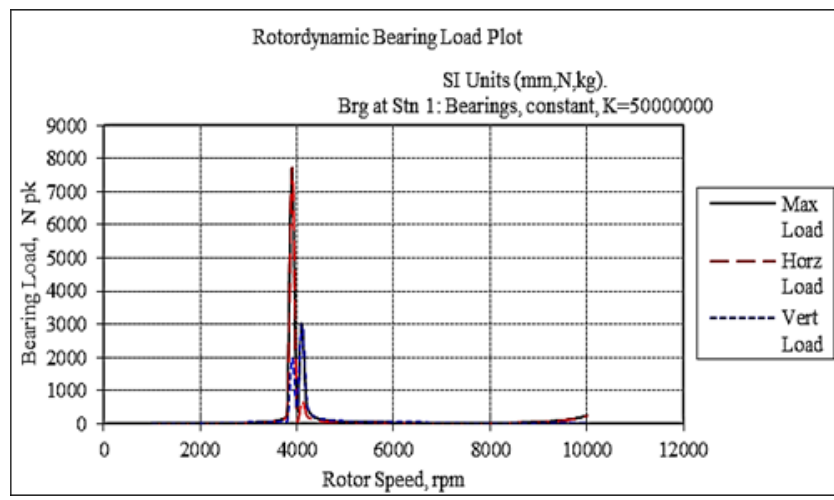

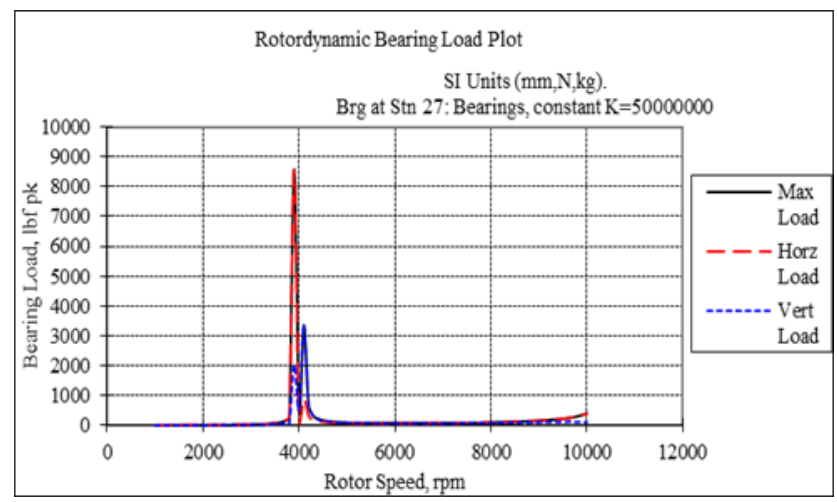

(b)

Figure 6. (a) Load on bearing at station 1 vs rotor speed; (b) Load on bearing at station 27 vs rotor speed

\subsubsection{Effect of Shaft (Steel) Diameter on Critical Speeds, Vibration in Velocity and Load of Bearings Due to Imbalance}

The first iteration is an analysis of the diameter of the shaft. The variation is from $80 \mathrm{~mm}$ to $120 \mathrm{~mm}$ for shaft diameter in order to understand the behavior of the rotor on critical speeds and rate of failure. For the 80mm diameter, the response at station 11 due to imbalance, as well as the dynamic load on station 1 and station 27 bearings are shown in Figure 2.

It is clearly observed from Figure 7(a), 7(b), and 7(c), that for $80 \mathrm{~mm}$ diameter of the shaft, the velocity at the $1^{\text {st }} \mathrm{critical}$ speed is vibrating at $0.021 \mathrm{~m} / \mathrm{s}$ at $2900 \mathrm{cpm}$ in the horizontal direction and $0.026 \mathrm{~m} / \mathrm{s}$ in $3100 \mathrm{cpm}$ in the vertical direction. At the $2^{\text {nd }}$ critical speed, the velocity is found to be increased to $0.032 \mathrm{~m} / \mathrm{s}$ at $9000 \mathrm{cpm}$ and 0.032 at $10000 \mathrm{cpm}$, which is much more than the maximum speed of 5000rpm. The failure rate is high at the $2^{\text {nd }}$ critical speed compared to the $1^{\text {st }} \mathrm{critical}$ speed and the resonance occurs well before at $2900 \mathrm{cpm}$ compared to the referred rotor model. Hence, early failure of the system is encountered. The vibration of the rotor and the load acting on bearings due to imbalance is shown in Table 4 .

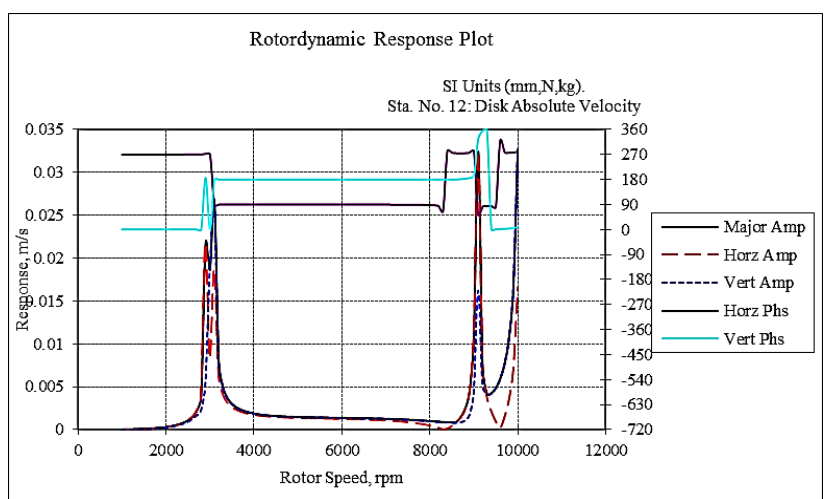

(a)

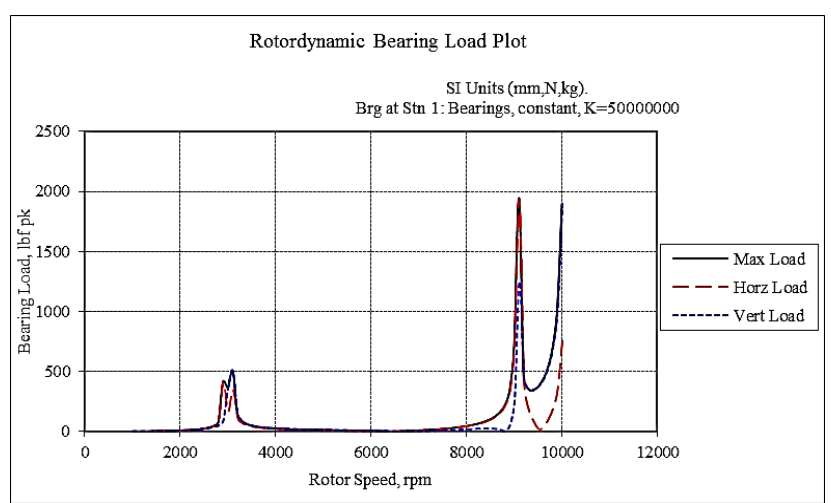

(b)

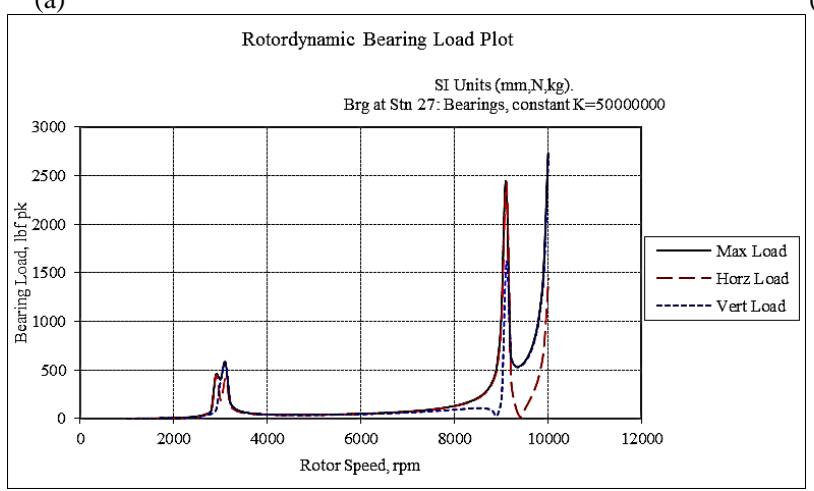

(c)

Figure 7. (a)Velocity response plot at station 11 vs rotor speed; (b) Load on bearings at station 1 vs rotor speed;

(c) Load on bearings at station 27 vs rotor speed 
Table 4. Velocity at station 11 and Load acting on stations 1 and station 27

\begin{tabular}{|c|c|c|c|c|}
\hline \multicolumn{2}{|c|}{ Imbalance results } & \multirow{2}{*}{ Stations } & \multicolumn{2}{|c|}{ Radial directions } \\
\hline S.No. & Parameters & & Horz Amp $(\mathrm{m} / \mathrm{s})$ & Vert $\operatorname{Amp}(\mathrm{m} / \mathrm{s})$ \\
\hline 1 & Velocity & \multirow[b]{2}{*}{11} & $0.021 @ 2900$ & $0.026 @ 3100$ \\
\hline 2 & Velocity & & $0.032 @ 9100$ & $0.032 @ 10000$ \\
\hline S.No. & Parameters & & Horz $\mathrm{N}$ & Vert $\mathrm{N}$ \\
\hline \multirow{2}{*}{1} & \multirow{2}{*}{ Load at 1 st bearing } & \multirow{2}{*}{1} & 417 N @ 2900 & $505 \mathrm{~N} @ 3100$ \\
\hline & & & 1939 N @ 9100 & $1898 \mathrm{~N} @ 10000$ \\
\hline \multirow{2}{*}{2} & \multirow{2}{*}{ Load at 2 nd bearing } & \multirow{2}{*}{27} & 455 N@ 2900 & 578 N@3100 \\
\hline & & & 2425 N @9100 & $2727 \mathrm{~N} @ 10000$ \\
\hline
\end{tabular}

The $1^{\text {st }}$ critical speed is determined at $2900 \mathrm{cpm}$ from Figure 8(a), which is well before the referred rotor model [17]. The velocity is vibrating at higher limits but the load on bearings is low, hence the bearings are safe as per the rated dynamic load. The rotor achieved higher loads on bearings. The system should encounter vibrating at higher limits with the $2^{\text {nd }}$ critical speed, which is not acceptable. Similarly, for the $90 \mathrm{~mm}, 100 \mathrm{~mm}, 110 \mathrm{~mm}$ and $120 \mathrm{~mm}$ diameters, the graph represents the range of critical speeds, vibration velocity and load on bearings at the $1^{\text {st }}$ and $27^{\text {th }}$ stations as shown Figure 2 .

The critical speed is directly proportional to shaft diameter from Figure 8(a). For the rotor model with increased diameter, the critical speed also tends to increase from $2900 \mathrm{cpm}$ to $4585 \mathrm{cpm}$. Critical speeds shifted $25-30 \%$ above/below its operating speed of rotor are considered as safe operating conditions. The impact of vibrating velocity on shaft diameter is shown in Figure 8(b).

For the $100 \mathrm{~mm}$ diameter, the velocity increased drastically and was found to be serious at the $1^{\text {st }}$ critical speed as shown in Figure 8(b). The horizontal peak is governing compared to the vertical direction. For the $120 \mathrm{~mm}$ diameter, the $1^{\text {st }}$ critical speed is at $4585 \mathrm{cpm}$, which is safe and vibrating beyond the referred model. From Figure 8(c), the maximum peak load acts for the $100 \mathrm{~mm}$ diameter shaft in the horizontal direction; hence, for this shaft diameter, the velocity is vibrating at higher limits at the $1^{\text {st }}$ critical speed. To design a rotor, the series of trials is carried out with disk offset, disk configuration and load on disk.

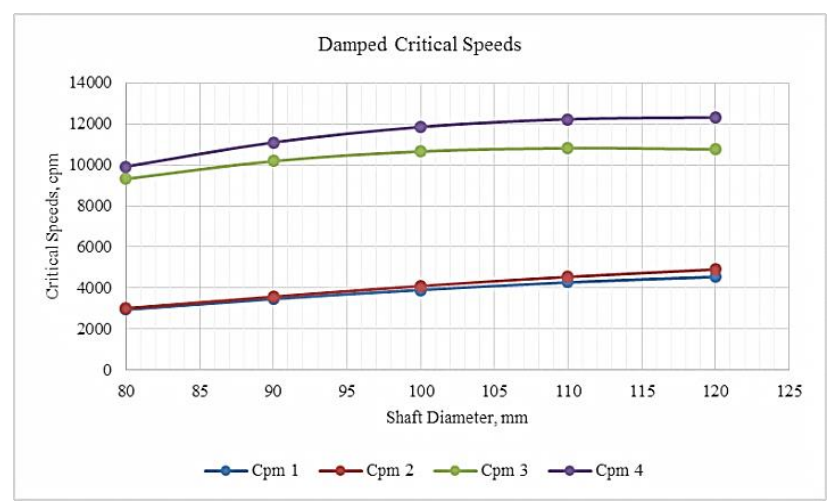

(a)

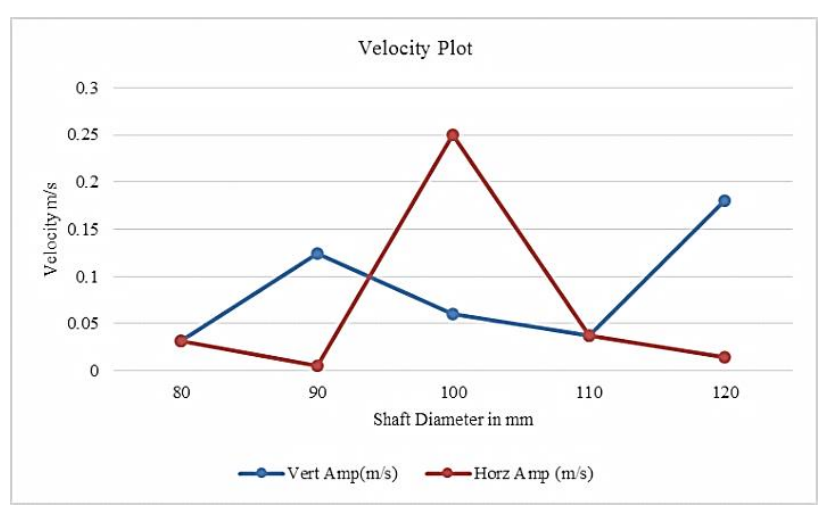

(b)

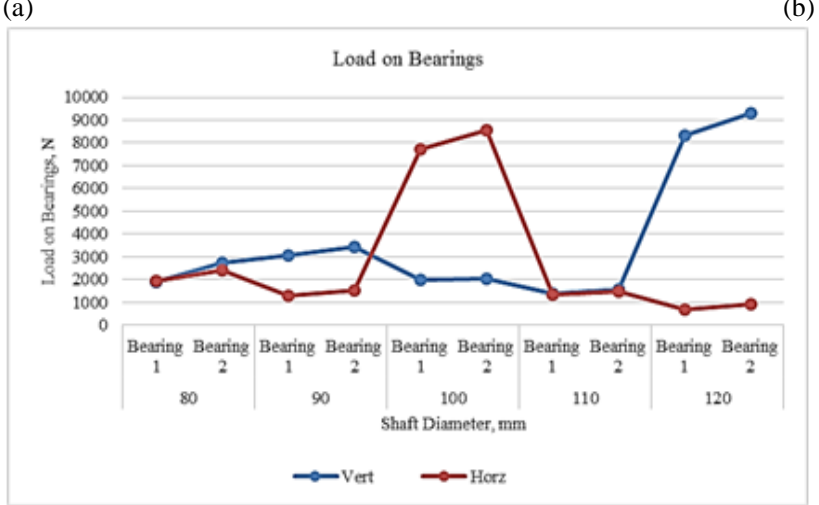

(c)

Figure 8. (a) Damped critical speed map vs shaft diameter; (b) Velocity vs shaft diameter; (c) Load on bearings at station 1 and station 27 vs shaft diameter 


\subsubsection{Effect of Disk Offset on Critical Speeds, Vibration in Velocity and Load of Bearings Due to Imbalance}

For the rotor model, the disk offset is $50 \mathrm{~mm}$ to check how far critical speeds vary compared to the referred model as shown in Figure 9(a).

In this trail, as shown in Figures 9(b) and 9(c), the critical speeds are vibrating close to the referred rotor model i.e. $3900 \mathrm{cpm}$ in the vertical direction. The shifting of critical speeds will not impact due to the disk offset on the model. Load on bearings is high at stations 127 in the vertical direction due to the $1^{\text {st }}$ disk offset to the right of $50 \mathrm{~mm}$. This is due to the shift of mass close to other disks of larger masses. The distribution of load is not at the centre of the disk as there is variation of sizes and mass of the disks. Hence, failure is increased at higher rates and considered unsafe at this condition. This trail is said to be highly unreliable as failure is early due to negligible decreases in critical speeds, vibrating with higher velocity and an increase in load on bearings.

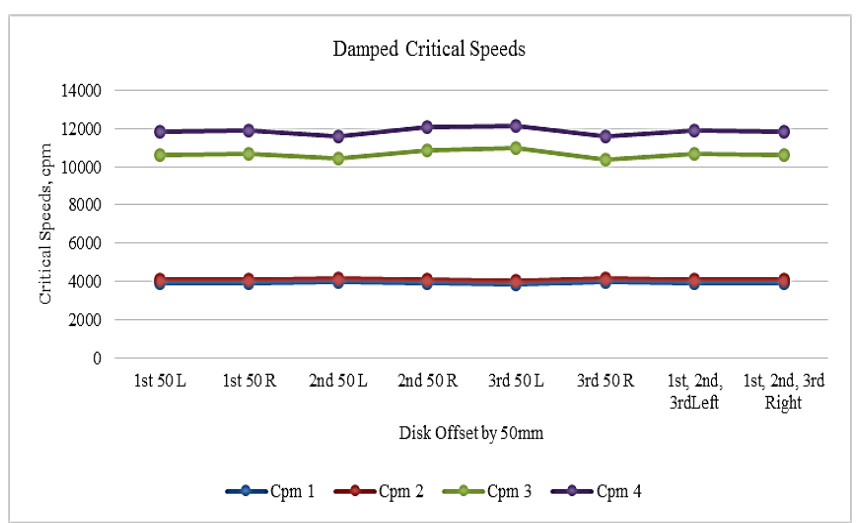

(a)

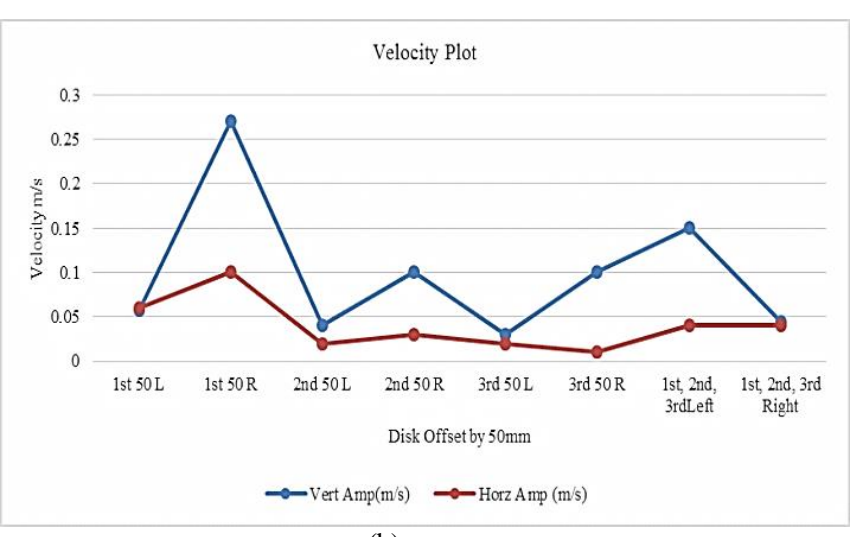

(b)

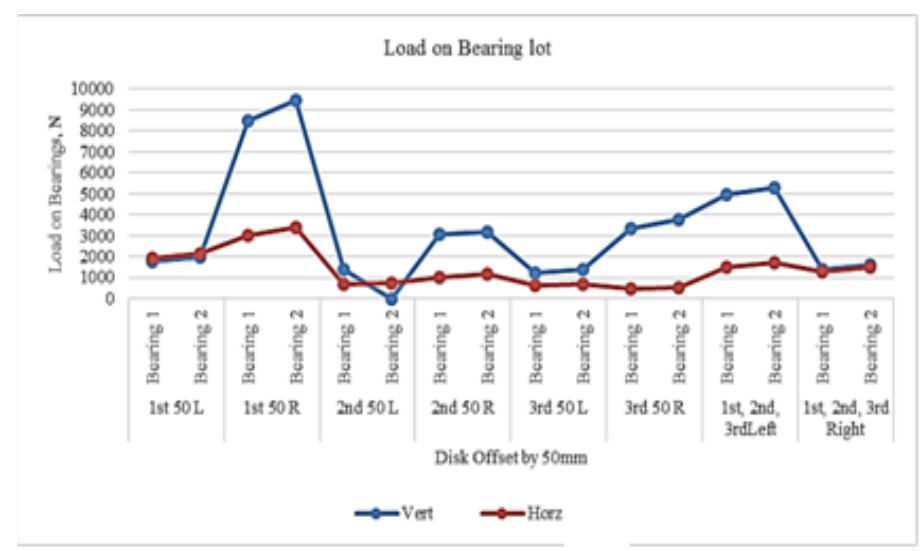

(c)

Figure 9. (a) Damped critical speed map vs disk offset; (b) Vibrating velocity vs disk offset; (c) Load on bearings for stations 1 and 27 vs disk offset

\subsubsection{Effect of Load on Disk on Critical Speeds, Vibration in Velocity and Load of Bearings Due to Imbalance}

In this trail, the load of $5 \mathrm{~kg}$ is introduced on the rotor model to check how critical speeds deviate from the referred model. The design of the rotor is said to be effective when critical speeds are shifted $30 \%$ above the operating speed. This trial decreases the critical speed. The critical speeds can be bypassed at a faster rate when they are vibrating away from operating speed. But in this case, a small mass of $5 \mathrm{~kg}$, which is $2 \%$ of total weight, has not really affected the critical speeds as they are vibrating close to the referred model as shown in Figure 10(a) in which the frequency is directly proportional to stiffness and indirectly proportional to mass.

For Figure 10(b), the vibrating velocity is high when the load is acting on disks 2 and 3. In Figure 10(c), the critical speeds of the rotor undergo failure when load is acting on the $2^{\text {nd }}$ and $3^{\text {rd }}$ disk, as distribution of mass is more on the $2^{\text {nd }}$ and $3^{\text {rd }}$ disks. The centre of gravity lies between disks 2 and 3. Because it bypasses the critical speeds, this method is suitable for rotor design. 


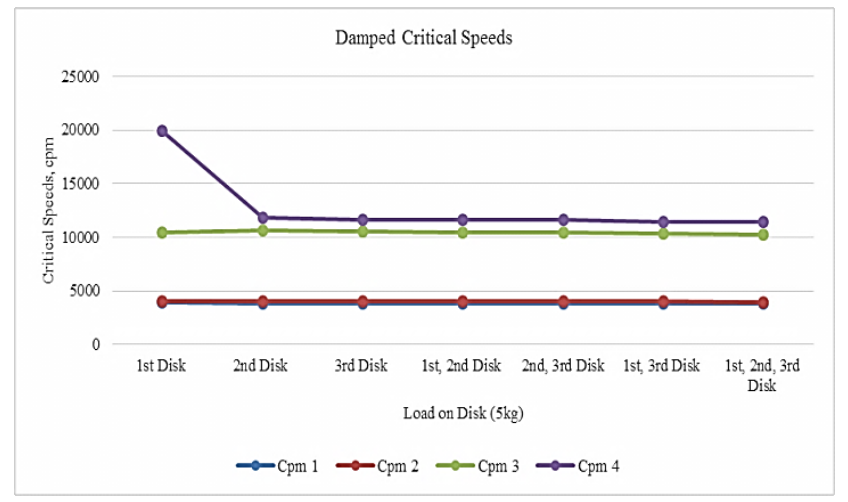

(a)

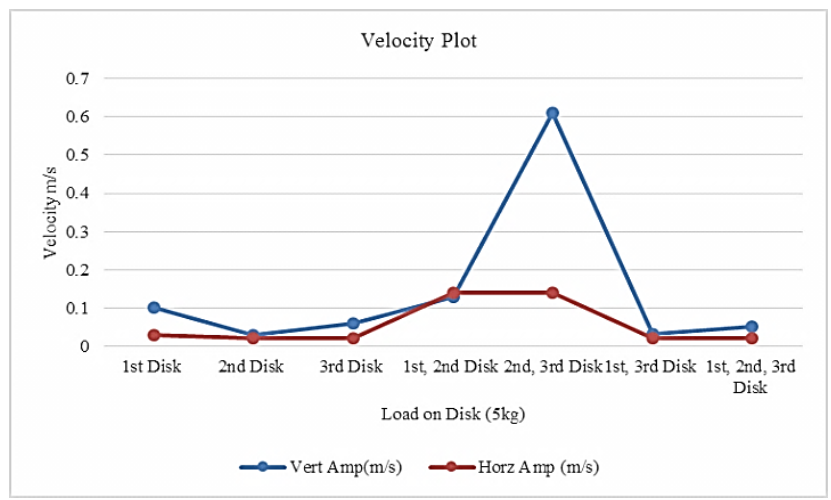

(b)

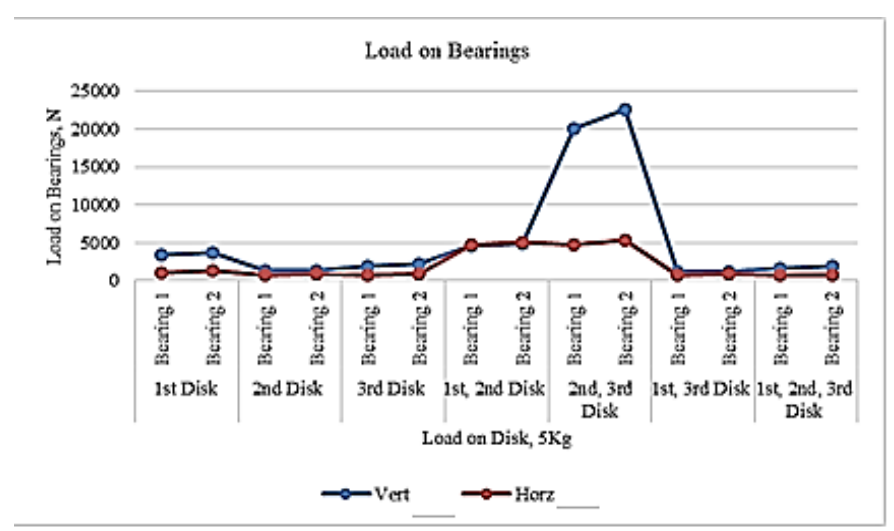

(c)

Figure 10. (a) Damped critical speed map vs load on disk; (b) Vibrating velocity vs load on disk;

(c) Load on bearings at station 1 and 27 vs load on disk

\subsubsection{Effect of Aluminum Disk on Critical Speeds, Vibration in Velocity and Load of Bearings Due to Imbalance}

The results are determined for disk configuration by introducing aluminium disk to the rotor model. The resonance is beyond the maximum speed and found to be safe and reliable compared to the referred model. The first critical speed is at $5265 \mathrm{cpm}$, which is $74 \%$ higher than the referred model's critical speed $(3900 \mathrm{cpm})$. This rotor model is designed for safe and high reliability. The idea of this trail is to clearly determine the impact of material damping on rotor model and design for safe operating conditions. Rotor vibration and load on bearings is high at all disk aluminium configurations as shown in Figures 11(a) and 11(b). Because of a shift of critical speeds, the rotor failure will occur beyond 5000cpm, which is considered to be safe as the rotor maximum speed is 5000rpm. The load on bearings is also considerably reduced from $8000 \mathrm{~N}$ (referred model) to $5000 \mathrm{~N}$ in the horizontal direction due to the aluminium disk on rotor. Figure 11 (c) shows the combination of AL disk on the rotor model.

\section{Balancing of Rotor Model in XLrotor}

Rotor imbalance is a condition that does not coincide with the geometric center and mass center. In reality, these centers never exactly coincide, but the main objective of this rotor model balancing is to reduce the imbalance to the extent that the residual unbalance does not affect machine life negatively. An attempt is made to balance the rotor of steel shaft with a steel disk (referred model) and steel shaft with an aluminum disk (critical speed shifted about 74\%). The balancing of the rotor model is carried out to the steel shaft with the steel disk and the steel shaft with the aluminum disk. The following shows balancing of the rotor.

\subsubsection{Balancing of Rotor Steel Shaft with Steel Disk (Referred Rotor)}

The rotor is balanced for a series of trails. For this configuration of rotor, the mass is distributed on disks 1,2 , and 3. The location of mass is at $0^{\circ}$ for disk $2,180^{\circ}$ for disk 1 and $180^{\circ}$ for disk 3 in order to balance the rotor for $200 \mathrm{gm}-\mathrm{mm}$. Table 5 shows the mass distributed on disks 1,2 , and 3 . 


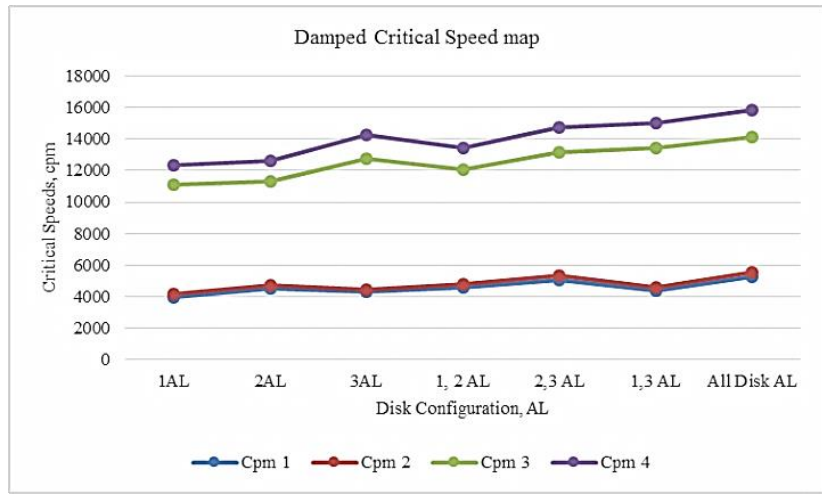

(a)

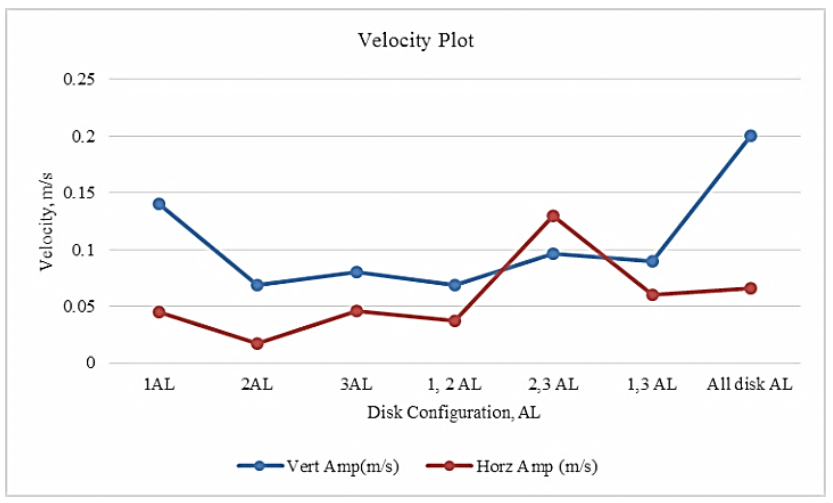

(b)

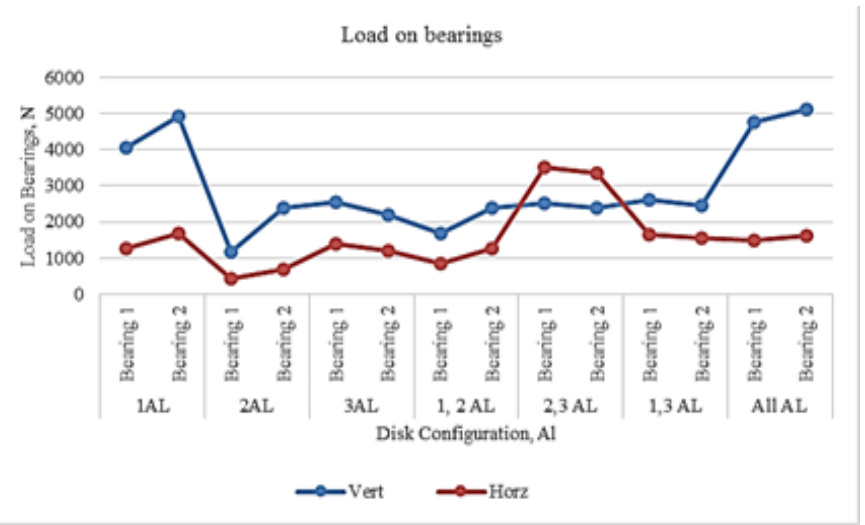

(c)

Figure 11. (a) Damped critical speed map vs disk configuration, AL; (b) Vibrating velocity vs disk configuration, AL;

(c) Load on bearings at stations 1 and 27 vs disk configuration, AL

Table 5 shows the combination of mass on disks. This is one of the corrective methods to minimize effect of vibration at resonance for the referred model vibrating at $3900 \mathrm{cpm}$. The amplitude is reduced from $0.25 \mathrm{~m} / \mathrm{s}$ (from Figure 5) to $0.0019 \mathrm{~m} / \mathrm{s}$ for disk $1,0.0032 \mathrm{~m} / \mathrm{s}$ for disk 2 , and $0.0024 \mathrm{~m} / \mathrm{s}$ for disk 3 for the referred model. The load on bearings is also considerably reduced to $100 \mathrm{~N}$ and $101 \mathrm{~N}$ at station 1 and 27, respectively, as shown in Figure 2. The results obtained are designed for reduced vibration levels as shown in Figures 12(a), 12(b), and 12(c).

Table 5. Disk location, vibration velocity \& load on bearings due to imbalance

\begin{tabular}{|c|c|c|c|c|c|}
\hline Location Disk & Unbalance (gm-mm) & Vibration Velocity $(\mathrm{m} / \mathrm{s})$ & Direction & Station 1 Load $(\mathrm{N})$ & Station $27 \operatorname{Load}(\mathrm{N})$ \\
\hline 1 & 110 & 0.006 & \multirow{3}{*}{ Radial } & \multirow{3}{*}{316} & \multirow{3}{*}{345} \\
\hline 2 & 200 & 0.01 & & & \\
\hline 3 & 150 & 0.008 & & & \\
\hline 1 & 112 & 0.0041 & \multirow{3}{*}{ Radial } & \multirow{3}{*}{205} & \multirow{3}{*}{221} \\
\hline 2 & 200 & 0.0066 & & & \\
\hline 3 & 152 & 0.0053 & & & \\
\hline 1 & 114 & 0.0026 & \multirow{3}{*}{ Radial } & \multirow{3}{*}{142} & \multirow{3}{*}{148} \\
\hline 2 & 200 & 0.0045 & & & \\
\hline 3 & 154 & 0.0034 & & & \\
\hline 1 & 115 & 0.0023 & \multirow{3}{*}{ Radial } & \multirow{3}{*}{121} & \multirow{3}{*}{125} \\
\hline 2 & 200 & 0.0039 & & & \\
\hline 3 & 155 & 0.0029 & & & \\
\hline 1 & 116 & 0.0019 & \multirow{3}{*}{ Radial } & \multirow{3}{*}{100} & \multirow{3}{*}{101} \\
\hline 2 & 200 & 0.0032 & & & \\
\hline 3 & 156 & 0.0024 & & & \\
\hline 1 & 118 & 0.0025 & \multirow{3}{*}{ Radial } & \multirow{3}{*}{130} & \multirow{3}{*}{152} \\
\hline 2 & 200 & 0.0041 & & & \\
\hline 3 & 158 & 0.0034 & & & \\
\hline 1 & 120 & 0.0048 & \multirow{3}{*}{ Radial } & \multirow{3}{*}{242} & \multirow{3}{*}{276} \\
\hline 2 & 200 & 0.0077 & & & \\
\hline 3 & 160 & 0.0063 & & & \\
\hline
\end{tabular}



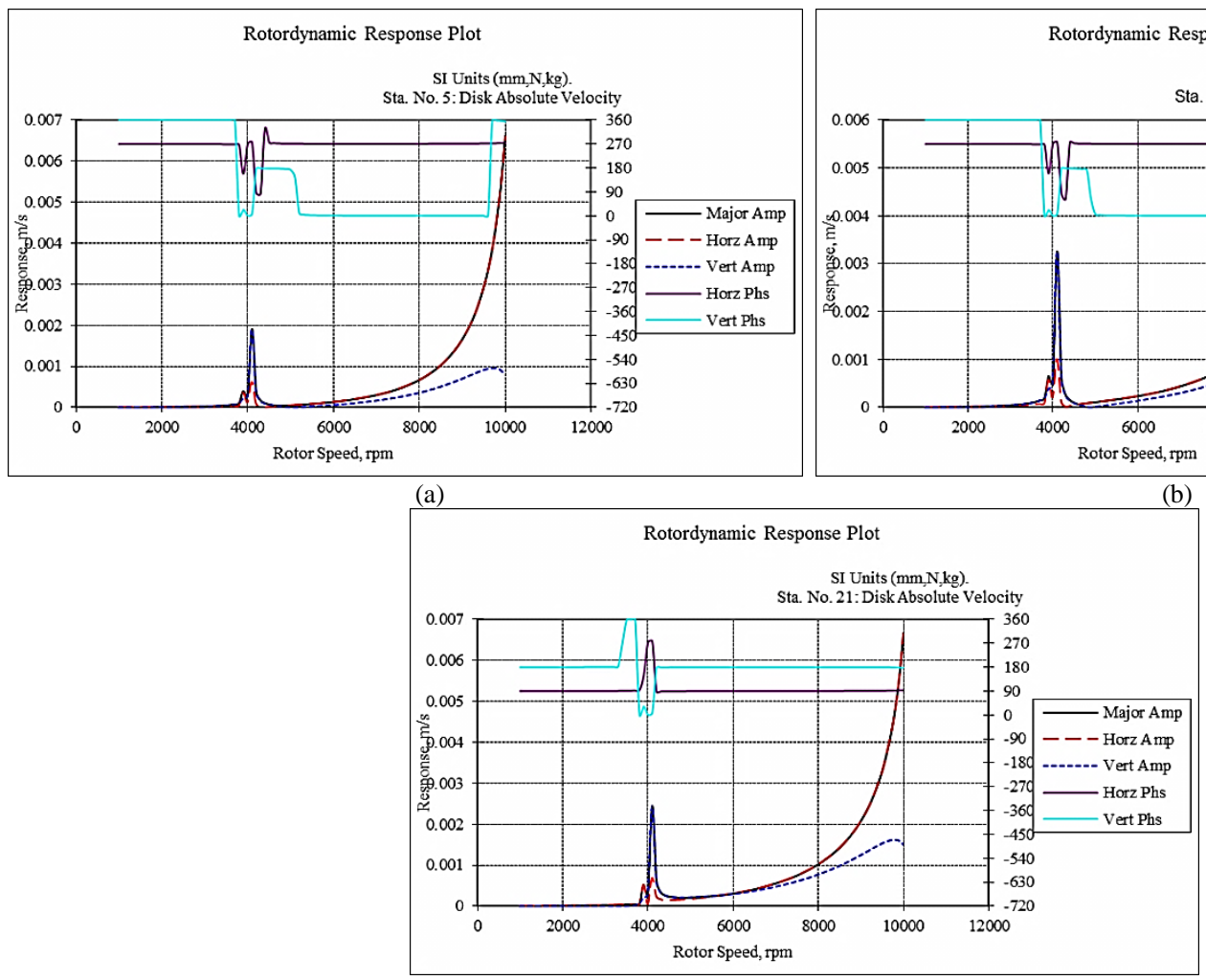

(c)

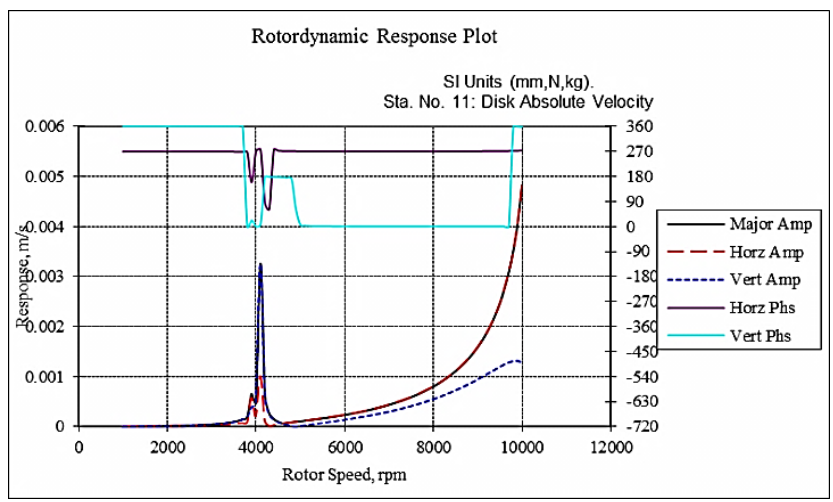

(b)

Figure 12. (a) Vibration velocity at Disk 1 (Station 5); (b) Vibration velocity at Disk 2 (Station 11); (c) Vibration velocity at Disk 3 (Station 21 )

\subsubsection{Balancing of Rotor Steel Shaft with Aluminum Disk (Optimized Rotor)}

In this trail, the balancing of the rotor is analyzed for the optimized rotor steel shaft with aluminum disk. This rotor is designed for safe and highly reliable operating conditions compared to the referred model, and critical speeds have shifted to $5265 \mathrm{cpm}$ from $3900 \mathrm{cpm}$. The rotor balancing mass in gm-mm is as shown in Table 6

\begin{tabular}{|c|c|c|c|c|c|}
\hline Location Disk & Unbalance (gm-mm) & Vibration Velocity $(\mathrm{m} / \mathrm{s})$ & Direction & Station $1 \operatorname{Load}(\mathrm{N})$ & Station $27 \operatorname{Load}(\mathrm{N})$ \\
\hline 1 & 116 & 0.0044 & \multirow{3}{*}{ Radial } & \multirow{3}{*}{177} & \multirow{3}{*}{171} \\
\hline 2 & 200 & 0.0075 & & & \\
\hline 3 & 156 & 0.0056 & & & \\
\hline 1 & 118 & 0.0028 & \multirow{3}{*}{ Radial } & \multirow{3}{*}{110} & \multirow{3}{*}{99} \\
\hline 2 & 200 & 0.0048 & & & \\
\hline 3 & 158 & 0.0035 & & & \\
\hline 1 & 120 & 0.0012 & \multirow{3}{*}{ Radial } & \multirow{3}{*}{44} & \multirow{3}{*}{28} \\
\hline 2 & 200 & 0.0021 & & & \\
\hline 3 & 160 & 0.0013 & & & \\
\hline 1 & 122 & 0.0017 & \multirow{3}{*}{ Radial } & \multirow{3}{*}{60} & \multirow{3}{*}{46} \\
\hline 2 & 200 & 0.0028 & & & \\
\hline 3 & 162 & 0.0019 & & & \\
\hline 1 & 124 & 0.0023 & \multirow{3}{*}{ Radial } & \multirow{3}{*}{81} & \multirow{3}{*}{69} \\
\hline 2 & 200 & 0.0037 & & & \\
\hline 3 & 164 & 0.0027 & & & \\
\hline
\end{tabular}

For this rotor, the vibration is reduced from $0.20 \mathrm{~m} / \mathrm{s}$ (from Figure $11(\mathrm{~b})$ ) to $0.0012 \mathrm{~m} / \mathrm{s}$, disk 2 at $0.0021 \mathrm{~m} / \mathrm{s}$, and disk 3 at $0.0013 \mathrm{~m} / \mathrm{s}$ as shown in Figures $13(\mathrm{a}), 13(\mathrm{~b})$, and 13(c), which is well within the limits of vibration severity criteria ISO standards. The load on bearings has considerably reduced to $44 \mathrm{~N}$ and $28 \mathrm{~N}$. The resonance will occur at $5265 \mathrm{cpm}$, which vibrates at a lower velocity. This is considered as a safe design for the model. 

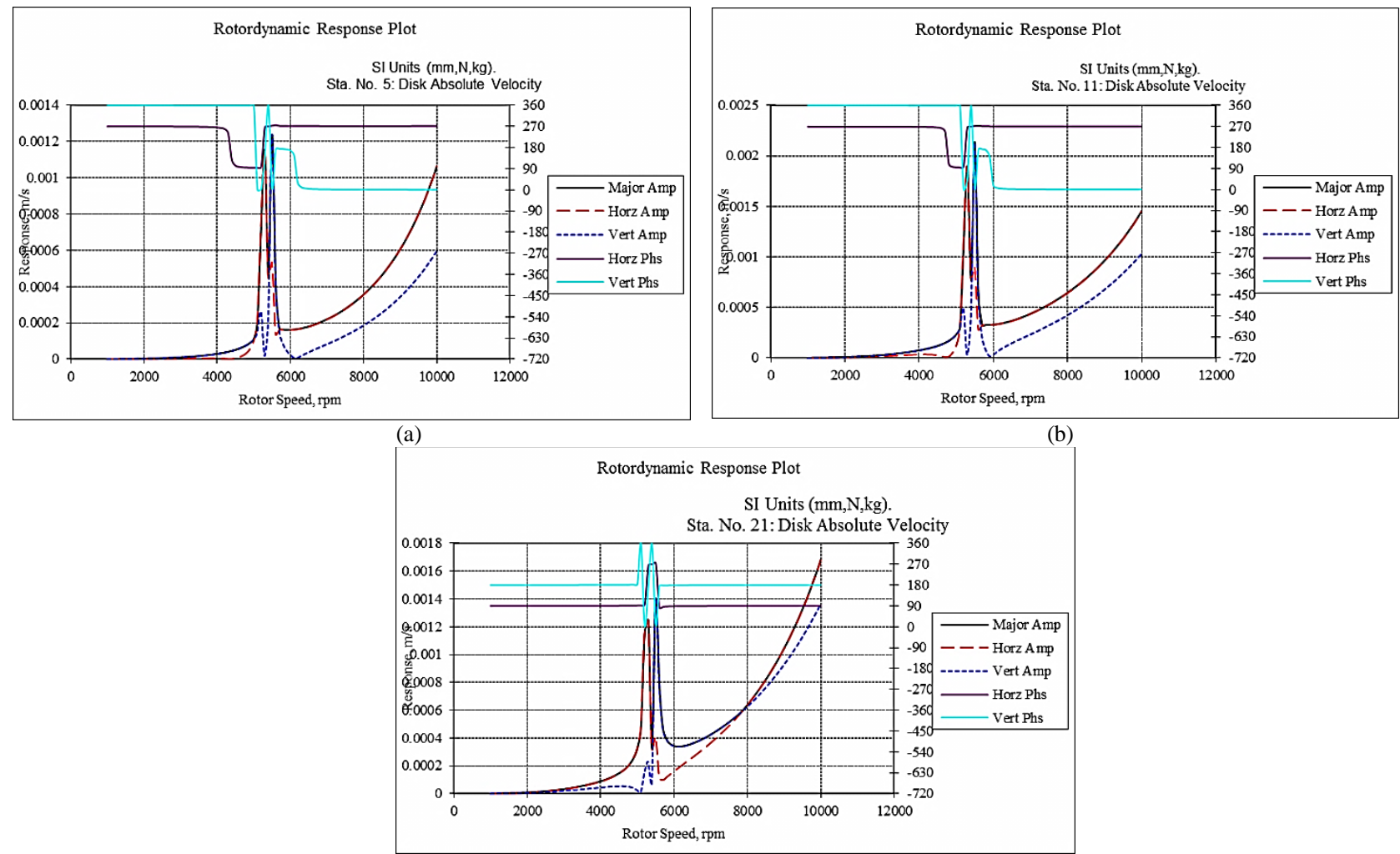

(c)

Figure 13. (a) Vibration velocity at disk 1 (Station 5); (b) Vibration velocity at disk 2(Station 11); (c) Vibration velocity at disk 3 (Station 21 )

\section{Applications of Rotors}

Rotors are used in industrial turbo engines and refrigeration compressors [12].

\section{Conclusions}

The complete rotordynamic analysis is designed for high reliability and safe operating conditions. The main aim of this study is to re-design the rotor, to shift critical speeds beyond its maximum speed, and to analyze the effect on vibration of rotor and load on bearings due to imbalance. The sensitivity analysis of the rotor is carried out on the diameter of the shaft, disk configuration, disk offset and disk load. The effect of aluminum disk on the rotor shows safe operating and high reliability compared to the referred steel disk rotor model. The following results are obtained:

1) Critical speeds are increased from $2953 \mathrm{cpm}$ to $4548 \mathrm{cpm}$ with an increase in diameter of the shaft from $80 \mathrm{~mm}$ to $120 \mathrm{~mm}$. The vibration and load on bearings is critical for the $100 \mathrm{~mm}$ shaft diameter.

2) Due to disk offset of $50 \mathrm{~mm}$ of all disks, the critical speeds are vibrating close to $2 \%$ of 3900 (referred model critical speed), which is negligible and thus, load on bearings is not decreased. Hence, this model is considered to be unreliable.

3) At an increase in load on disk of $5 \mathrm{~kg}$ on all disks, which is $2 \%$ of the total load acting on the rotor, the critical speeds are decreased from $3887 \mathrm{cpm}$ to $3782 \mathrm{cpm}$. Similarly, the velocity and load on bearings considerably increased due to the added mass on disks.

4) With change in the material from steel disk to aluminum disks, the critical speeds increased from $3900 \mathrm{cpm}$ to $5265 \mathrm{cpm}$, and so this model is designed for high reliability. The velocity and failure of bearings occurs at $5265 \mathrm{cpm}$ and this optimized model is safe as it is designed for maximum speed.

5) The rotor is balanced for 2 cases:

- Referred model steel shaft with steel disk: The vibrating velocity is reduced from $0.25 \mathrm{~m} / \mathrm{s}$ to $0.0019 \mathrm{~m} / \mathrm{s}$. The load on 
bearings at station 1 is decreased from $8000 \mathrm{~N}$ to $100 \mathrm{~N}$ and for station 27 from $8500 \mathrm{~N}$ to $101 \mathrm{~N}$. The amplitude and bearing load are reduced considerably.

- Optimized model steel shaft with aluminum disk: The vibrating velocity is reduced from $0.20 \mathrm{~m} / \mathrm{s}$ to $0.0012 \mathrm{~m} / \mathrm{s}$. The load on bearings at station 1 decreased from $4900 \mathrm{~N}$ to $44 \mathrm{~N}$ and at station 27 from $5200 \mathrm{~N}$ to $28 \mathrm{~N}$. The amplitude is reduced drastically and the load on bearings is dramatically decreased, which is safe as per the vibration severity criteria ISO standards. This model is optimized for high reliability.

\section{Acknowledgements}

The authors of this papers would like to thank all of the authors of referred papers.

\section{References}

1. M. Nakhaeinejad and S. Ganeriwala, "Rotordynamic Analysis using XLrotor," Spectra Quest Inc., 2008

2. N. G. Wagner, "Reliable Rotor Dynamic Design of High-Pressure Compressors based on Test Rig Data1," Journal of Engineering for Gas Turbines and Power, Vol. 123, No. 4, 2001

3. A. Gelin, J. -M. Pugnet, D. Bolusset, and P. Friez, "Experience in Full-Load Testing Natural Gas Centrifugal Compressors for Rotordynamic Improvements," Journal of Engineering for Gas Turbines and Power, Vol. 119, No. 4, pp. 934-941, 1997

4. E. A. Memmott, "Stability of Centrifugal Compressors by Applications of Tilt Pad Seals, Damper Bearings, and Shunt Holes," C432/070 IMechE., 1992

5. J. M. Sorokes, M. J. Kuzdzal, M. B. Sandberg, and G. M. Colby, "Recent Experiences in Full Load Full Pressure Shop Testing of a High Pressure Gas Injection Centrifugal Compressor," in Proceedings of the 23rd Turbomachinery Symposium, Texas A\&M University, College Station, 1994

6. R. Walker, S. Perinpanayagam, and I. K. Jennions, "Rotordynamic Faults: Recent Advances in Diagnosis and Prognosis," International Journal of Rotating Machinery, Vol. 2013, No. 5, pp. 12, 2013

7. E. J. Gunter, "Critical Speed Analysis of Offset Jeffcott Rotor using English and Metric Units," Fellow ASME, 2004

8. R. Tiwari, "A Brief History of Rotor Dynamics and Recent Trends," IIT Guwahati, 2017

9. B. T. Murphy, J. R. Kitzmiller, R. Zowarka, J. Hahne, and A. Walls, "Rotordynamics Design and Test Results for a Model Scale Compulsator Rotor," IEEE Transactions on Magnetics, Vol. 37, No. 1, pp. 310-313, 2001

10. A. H. Hishamet and M. R. Askari, "The Experimental Analysis of Vibration Monitoring in System Rotor Dynamic with Validate Results using Simulation Data," International Scholarly Research Network ISRN Mechanical Engineering, No. 11, 2012

11. S. Y. Yoon, Z. L. Lin, and P. E. Allaire, "Introduction to Rotor Dynamics," Control of Surge in Centrifugal Compressors by Active Magnetic Bearings, Advances in Industrial Control, Springer-Verlag London, 2013

12. E. Malcolm. Leader, P. E., "Using Rotordynamics to Solve Serious Machinery Vibration Problems," Applied Machinery Dynamics Co.P.O. BOX 157 Dickinson, TX7539MLeader@RotorBearingDynamics.COM

13. P. M. Todorović, B. Jeremic, I. Macuzic, A. Brkovic, and U. Proso, "Vibration Analysis of Cracked Rotor During Run-up" Tribology in Industry, Vol. 30, No. 1\&2, 2008

14. V. M. S. Hussain, "Reliability Modeling of Rotary Systems Subjected to Imbalance," International Journal of Performability Engineering, Vol. 9, No. 4, pp. 423-432, July 2013

15. M. Lalanneand and G. Ferraris, "Rotordynamics Prediction in Engineering," 2nd Edition, John Wiley and Sons, pp. 125-132, 1997

16. Vastard Veersh, "Solid Model Rotordynamics," White Paper Solid Model Rotor Dynamics, QuEST Global Services, 2012

17. T. Dimond, J. Chaudhry, M. Wagner, F. He, J. Cao, and P. E. Allaire, "Rotordynamic Analysis of a Rotor-Disk System Including a Synchronously Reduced Modal Truncation Method," in Proceedings of the ASME 2013 International Design Engineering Technical Conferences \& Computers and Information in Engineering Conference IDETC/CIE, 2013 